\title{
CLAVES FUNDAMENTALES EN TERAPIA DE PAREJA: UNA GUÍA DE NAVEGACIÓN PARA LA PRÁCTICA
}

\section{FUNDAMENTAL KEYS IN COUPLE THERAPY: A NAVIGATION GUIDE FOR PRACTICE}

\author{
Adrián Montesano \\ Departamento de Personalidad, Evaluación y Tratamientos Psicológicos, \\ Universidad de Barcelona, Barcelona, España \\ Escuela de Psicología, Universidad de Minho, Braga, Portugal
}

Cómo referenciar este artículo/How to reference this article:

Montesano, A. (2015). Claves fundamentales en terapia de pareja: Una guía de navegación para la práctica. Revista de Psicoterapia, 26(102), 161-174.

\section{Resumen}

En las últimas décadas, la terapia de pareja ha experimentado una creciente demanda social. La mayoría de los psicoterapeutas la ejercen en algún momento de su trayectoria profesional y muchos de ellos la practican regularmente. Sin embargo, la terapia de pareja es una de las modalidades más dificiles que existen, con un alto índice de fracaso, que requiere de una formación especifica muchas veces inexistente. En el presente texto se ofrecen algunas coordenadas clave para orientar la práctica clínica. En concreto, (a) se delinean los rasgos distintivos de la terapia de pareja como modalidad terapéutica independiente, (b) se comenta su evolución revisando los principales resultados de la investigación, y (c) se especifican siete coordenadas fundamentales para el desarrollo de una buena práctica.

Palabras clave: terapia de pareja, psicoterapia, psicoterapeutas, técnicas de intervención, terapia familiar

\begin{abstract}
In the last decades, couple therapy has experienced a growing social demand. Most psychotherapists practice it at some point of their careers and many of them do it regularly. However, couple therapy is one of the most difficult existing modalities, showing a high rate of failure, and that requires specific training which is lacking. In this paper I present some fundamental keys for guiding clinical practice. In particular, I (a) outline the distinctive features of couple therapy as an independent therapeutic modality; (b) comment on its evolution, reviewing main research outcomes, and (c) specify seven fundamental coordinates for the development of good practices.
\end{abstract}

Keywords: couple therapy, psychotherapy, psychotherapists, intervention techniques, family therapy

Fecha de recepción: 24 de septiembre de 2015. Fecha de aceptación: 29 de octubre de 2015.

Correspondencia sobre este artículo:

E-mail: adrianmontesano@gmail.com

Dirección postal: Departament de Personalitat, Avaluació i Tractaments Psicològics, Facultad de Psicologia, Universitat de Barcelona, Passeig Vall d'Hebron, 171, 08035.

(C) 2015 Revista de Psicoterapia 
La Terapia de Pareja (TP) ha sido definida como una de las modalidades de psicoterapia más difíciles que existen. La complejidad de variables involucradas, las dosis elevadas de conflictividad interpersonal que comporta en la mayoría de las ocasiones, y los múltiples componentes de la relación terapéutica, imponen un ritmo trepidante no apto para terapeutas inexpertos. Es una modalidad terapéutica que requiere, sin duda, de una especialización profesional, dadas sus particularidades en el setting y en el desarrollo del proceso terapéutico. El psicoterapeuta que quiera atender parejas de forma efectiva necesita conocer algunas reglas básicas que son diferentes a otras modalidades terapéuticas pero que resultan ser claves para la formulación de caso y el desarrollo de la intervención. Un terapeuta acostumbrado a la terapia individual precisará suspender sus guiones habituales de entrevista y acoplarse al hecho de contar en la sala con los dos miembros de la pareja (a veces incluso con un tercero no presente), con su relación, con un co-terapeuta y, en condiciones ideales, con un equipo que le acompaña. Frente a la decisión de aventurarse en este entramado relacional, el terapeuta necesita equiparse con mapas para navegar durante las subidas y bajadas del proceso terapéutico.

El principal objetivo de este artículo es ofrecer algunas coordenadas clave de navegación para orientar la práctica clínica. Primero se delinean los rasgos distintivos de la TP señalando los riesgos y precauciones que se deben tomar. Después se revisa su evolución atendiendo tanto al desarrollo teórico como a los principales resultados de la investigación. Por último, se especifican siete coordenadas fundamentales para el desarrollo de una buena práctica.

\section{RASGOS DISTINTIVOS DE LA TERAPIA DE PAREJA}

Ciertamente, la psicoterapia con parejas dista mucho de parecerse a la terapia individual y requiere de un entrenamiento adecuado que asegure la competencia del terapeuta. Es una realidad, no sólo en nuestro país, que la mayoría de los psicoterapeutas no recibe en su entrenamiento ninguna formación específica en TP, ni siquiera en la formación oficial de posgrado. No obstante, muchos psicoterapeutas, a lo largo de su trayectoria profesional, cometen la temeridad de aceptar casos de pareja. No hay que ser muy astuto para adivinar que en muchos de los casos, tales atrevimientos han desembocado en fracasos y frustraciones de ambos, de la pareja que consulta y del terapeuta. En la mejor de las situaciones, la pareja decidirá abandonar el tratamiento y buscar otra alternativa, en el peor, las intervenciones mal orquestadas pueden empeorar la situación de la pareja consultante pues, al fin y al cabo, la psicoterapia también puede tener efectos secundarios nocivos.

Pareciera sin embargo que la TP resulta una modalidad muy atractiva para los psicólogos y psicoterapeutas. Por ejemplo, en el estudio más exhaustivo de la práctica de la psicoterapia en EEUU se encontró que cerca del 75\% de los terapeutas visitan parejas en su práctica privada (Orlinsky y Roneastad, 2005). Aunque se desconocen los datos, en España es muy probable que todos los terapeutas de la práctica privada se vean atraídos en algún momento para atender una demanda de 
pareja y muchos la practiquen con cierta regularidad. Las probabilidades son altas porque, enfatizado sea de paso, el ámbito privado es prácticamente al único servicio al que pueden acceder las parejas, pues el malestar relacional es una de las áreas más marginadas por los servicios asistenciales. En cualquier caso, resulta un misterio saber dónde y cómo esta gran cantidad de psicoterapeutas han recibido su entrenamiento, pues como se ha señalado antes, la TP no tiene lugar ni en la formación de pregrado ni en la gran mayoría de los programas oficiales de formación de psicoterapeutas de nuestro país y tan poco en muchos otros países.

Desde el punto de vista de las parejas, esto supone un auténtico problema, pues la probabilidad de encontrar un profesional específicamente formado es sumamente baja. Dada la falta de regulación de la profesión de psicoterapeuta, el hecho de que un centro anuncie la TP en su cartera de servicios no garantiza en absoluto la especialización de los profesionales. En vista de todas estas cuestiones no es de extrañar que fuese precisamente la TP la única forma de terapia que recibió una mala valoración en la famosa encuesta de clientes de psicoterapia (Seligman, 1995).

\section{¿Por qué la terapia de pareja es pues tan compleja y diferente de la terapia individual?}

Las razones son muchas. En el primer párrafo de este texto ya hemos avanzado las más salientes. La primera gran dificultad tiene que ver con la naturaleza diferente de la alianza terapéutica. Incluso el terapeuta más habilidoso en generar una buena alianza en terapia individual, puede verse catapultado al fracaso en cuestión de segundos en una TP si, al querer forjar una buena relación con uno de los miembros, se gana la enemistad del otro miembro de la pareja. Aunque mantener un equilibrio en la alianza con cada miembro parezca de sentido común, la verdad que en la práctica clínica es una tarea más dificultosa de lo que parece, y muchos terapeutas quedan entrampados en triangulaciones que minan cualquier posibilidad de éxito de la terapia. Los terapeutas familiares conocen bien este aspecto, pero no así los terapeutas formados en terapia individual, cualquiera que sea el modelo de referencia.

Pero además, más allá de evitar trampas triangulatorias, el terapeuta debe prestar mucha atención a otro componente específico de la alianza en el contexto de una TP: la alianza intra-sistema. El terapeuta tiene que ser consciente de que la principal relación que vehiculiza el cambio no es con él o ella, sino la de la propia pareja. Por tanto, el foco terapéutico debe privilegiar la relación de pareja sobre la terapéutica. En esta diferencia radica una de las ventajas principales de la TP: permite trabajar con el contexto en el que se enmarcan los problemas y utilizarlo como fuente de recursos para la intervención.

Otra de las características diferenciadoras de la TP es que las sesiones están frecuentemente cargadas de elevadas dosis de tensión, conflicto y emociones a flor de piel. Perder el control de la sesión durante un breve lapso de tiempo puede resultar en que la pareja inicie una fuerte discusión, y que la voz del terapeuta se vea 
difuminada por los gritos y reproches de la pareja. En no pocas ocasiones se puede llegar a una situación tragicómica: la pareja se pasa la sesión entera enganchada en el conflicto, y el terapeuta se pregunta, desde la barrera, cómo es posible que alguien esté dispuesto a pagarle para que les observe discutir. Es verdad que en algunas situaciones clínicas el proceso puede exigir la intensificación del conflicto, pero siempre dentro de una estrategia terapéutica, no como consecuencia de la falta de estructura.

Así pues, quedarte sin estructura en el contexto de una TP puede tener un resultado fatal, y por tanto, darse tiempo para pensar en el transcurso de la sesión, es un lujo que uno no puede permitirse en la mayoría de las ocasiones. Existen procedimientos para salvar estos escollos, como trabajar en co-terapia, utilizar guiones, hacer reuniones antes o después de la sesión; pero en cualquiera de los casos un cierto nivel de tolerancia a la tensión emocional y la conflictividad, resulta ser una herramienta indispensable para sobrevivir como terapeuta de pareja.

Esta tolerancia al conflicto será también un punto de apoyo frente al hecho de que en la mayoría de las ocasiones la TP comienza con la amenaza de que la pareja está a punto de romperse. Esto es un poco, salvando las distancias, como si cada cliente de terapia individual comenzase la terapia diciendo que quiere suicidarse $o$ hacerse daño a sí mismo, cosa que ocurre pero con una frecuencia mucho más baja. En cambio, en la TP la amenaza de ruptura está a la orden del día. Esto, sin duda, requiere de monitorizar el proceso de manera muy cuidadosa y sensible. Para ello, es preciso realizar una buena evaluación de la demanda y del nivel de crisis actual de la pareja para poder ajustar de forma adecuada la intervención. En la práctica esto puede requerir el tener que intervenir antes de poder recoger toda la información necesaria, hacer pactos especiales con la pareja, intensificar la frecuencia de las sesiones o incluso, en casos más extremos, contraindicar la TP si no se cambian las condiciones de la demanda. En la TP, a veces una retirada a tiempo es una victoria.

Quizás uno de los rasgos distintivos más peliagudos de la TP es la inevitabilidad de tener que afrontar dilemas morales sobre la naturaleza de las relaciones humanas. Temas tales como el amor, la muerte, el deseo, el rechazo, la ternura, la intimidad, el compromiso, el sexo, la (in)fidelidad, el maltrato, la violencia, la soledad, la responsabilidad, el crecimiento, y un sinfín, pueden suponer un desafío para el terapeuta en asegurar el descentramiento de sus propios valores. La posición de neutralidad en la TP, es más un mito que una realidad.

No es mi intención en este texto desalentar a los psicoterapeutas a practicar la TP ni disuadir a aquellos que se sientan atraídos por ejercerla. Muy al contrario, mi objetivo es invitar a investigadores y terapeutas a profundizar en el conocimiento sobre el fascinante mundo de las relaciones humanas. Para que la comunidad de conocimientos avance es importante tener una mínima orientación de donde se está y cuáles son los desafíos inminentes. Esperamos que los apartados subsiguientes ayuden al lector a situarse con respecto al estado de la TP en la actualidad y pueda contribuir con su propia práctica a evolucionar. 


\section{LA EVOLUCIÓN DE LA TERAPIA DE PAREJA}

La TP-incluida la terapia sexual como subespecialidad-ha experimentado un fuerte aumento de personas que solicitan ayuda profesional en los últimos años. Probablemente, esta demanda creciente se deba a los cambios socioculturales acontecidos desde finales del siglo XX. Las reformas en las normas legales y culturales sobre la separación y el divorcio, la apertura política y social hacia un rango más amplio de expresiones del amor, el compromiso, la intimidad y la sexualidad, así como la evolución en el reparto del poder económico y político entre hombres y mujeres, han dibujado un escenario particular de retos que desafían la madurez de la TP como modalidad psicoterapéutica.

La TP no ha estado ajena a estos cambios y ha experimentado una evolución notable en las últimas décadas, especialmente en cuanto a su desarrollo teórico. Gurman y Fraenkel (2002) han identificado cuatro grandes etapas en el desarrollo de la TP hasta su estado actual. La primera etapa (1930-1963), calificada de ateórica, estuvo basada en la asesoría matrimonial llevada a cabo por consejeros no expertos en psicoterapia ni en salud mental. La segunda (1930-1966), denominada experimentación psicoanalítica, estuvo determinada por la práctica clínica de algunos intrépidos psicoanalistas que se aventuraron a invitar a parejas al contexto terapéutico. El mayor desarrollo de la disciplina, sin embargo, se produjo durante la tercera etapa (1963-1985), con la incorporación de la terapia familiar sistémica y el influjo de autores destacados como Don Jackson, Virginia Satir, Murray Bowen y Jay Haley; y otros posteriores como Jonh Gottman o Susan Jonshon, por nombrar algunos.

En la actualidad, la TP se encuentra en una etapa de refinamiento teórico y de expansión de su aplicabilidad, fruto de la incorporación de otros modelos como el cognitivo conductual, la terapia focalizada en las emociones, la terapia narrativa o la terapia psicodinámica, así como de la influencia de la perspectiva feminista, y la sensibilidad a lo multicultural (Gurman, Lebow y Snyder, 2015).

En efecto, en las últimas décadas han acontecido a gran velocidad cambios culturales de gran significación para aquellos que se embarcan en la aventura de (man)tener una relación de pareja, y la TP se ha visto impelida a adaptarse a estas demandas cambiantes. En mi opinión, uno de los retos vigentes más significativos radica en pasar del discurso sobre "cómo ayudar a las parejas a mantener una buena relación" al discurso de "cómo reconocer la multiplicidad de formas en que es posible ser o no una pareja".

La evolución de la TP también ha producido una cantidad considerable de investigación en muchas vertientes y constructos teóricos diferentes, imposibles de abarcar en este trabajo. Vamos a repasar, brevemente, algunas lecciones a mi parecer ya aprendidas en el campo de la investigación en psicoterapia. 


\section{Qué dice la investigación}

Si bien es cierto que el nivel de desarrollo de la investigación alcanzado por la TP va siempre por detrás del logrado por la terapia individual, también es verdad que décadas de investigación la sitúan como una modalidad altamente útil y prometedora. En efecto, la eficacia de la TP ha sido constatada repetidamente en los últimos treinta años (véase Darwiche y de Roten, 2015; para una revisión reciente). Los resultados meta-analíticos han mostrado que mejora la satisfacción relacional después de la intervención, obteniendo tamaños del efecto que van de moderados a altos ( $d=.59$; Shadish y Baldwin, 2005; $d=.86$; Christensen et al., 2004).

En general, se puede afirmar que existe una evidencia amplia sobre su efectividad, no solo como intervención para demandas relacionales (separación, violencia, infidelidad, etc.), sino también cuando un miembro de la pareja presenta un problema médico, o algún tipo de trastorno psicológico como depresión, ansiedad, adicciones, o trastornos alimentarios, entre otros (Baucom, Belus, Adelman, Fischer, y Paprocki, 2014; Campo, 2014; Leff et al., 2000; Montesano, Feixas, Muñoz y Compañ, 2014; Selekman y Beyebach, 2013).

Así pues, la TP posee diferentes ámbitos de aplicación y ha conseguido consolidarse como una alternativa terapéutica eficaz incluso para ciertas problemáticas individuales. Frente a la terapia individual, cuenta con la ventajosa particularidad de que trabaja con el contexto relacional más significativo del cliente. Ello supone una fuente privilegiada de recursos terapéuticos que permite amplificar el repertorio técnico y ecologizar las intervenciones, de modo que se fomente el mantenimiento del cambio a largo plazo. Así, una de las ventajas de la TP radica en su capacidad de reducir simultáneamente la sintomatología y el estrés relacional. Ello la ha convertido en una alternativa de tratamiento preferente cuando a la sintomatología acompaña una elevada conflictividad conyugal. Sin embargo, lo que hace a la TP más atractiva es que, incluso en parejas que no muestran problemas relacionales, el camino hacia la recuperación a largo plazo sigue estando mediado por mecanismos interpersonales (Whisman et al., 2012).

A pesar de la gran evolución de la TP desde principios del siglo XX tanto a nivel teórico como empírico, es importante reconocer que se está lejos de tener un conocimiento completo de cómo, cuándo y por qué se produce el cambio terapéutico. Además, los resultados de la eficacia deben ser mejorados, no sólo por el hecho de necesitar más estudios en contextos naturalísticos, sino porque los datos indican que el 25-30\% de las parejas no se benefician de la terapia (Snyder y Halford, 2012), y que hasta un $45 \%$ de las que mejoran en el comienzo se deterioran en el seguimiento a dos años (Christensen et al., 2004). Desde este punto de vista la TP aparece como uno de los campos con mayor índice de fracaso, cuestión a la que hay que dar respuesta tanto desde la investigación como de la práctica.

La ya clásica guerra entre escuelas de psicoterapia también es visible en el terreno de la TP. Pero en mi opinión de una manera mucho más suave y difuminada que en el campo de la psicoterapia individual. Quizás esa sea una de las ventajas de 
ir por detrás de ella en el desarrollo de la investigación, pues permite sortear los caminos erráticos y dirigir la atención a producir en tierra más fértil. Aun así, en la literatura se percibe la tensión entre los partidarios de factores específicos y los defensores de los factores comunes en la construcción del conocimiento sobre la teoría del cambio. En mi visión, ya hay una clara evidencia a favor de la equidad entre los distintos modelos en términos de eficacia y utilidad. Por tanto, espero que no se derroche ni el esfuerzo, ni el dinero, ni la tinta en concluir que la sentencia del pájaro Dodo es totalmente aplicable al campo de la TP: todos ganan, todos tiene premio.

Mi apuesta sería que la literatura tanto académica como no académica se focalizase en asuntos más fructíferos que dilucidar quién es el mejor. Asuntos como por ejemplo, explorar la manera en que se puede articular una red asistencial de calidad que permita el fácil acceso a la parejas, diseñar el modo de introducir la TP en el currículo de la formación de psicoterapeutas a la vista de que muchos de ellos la practicarán a lo largo de sus carreras, o investigar sobre cómo se puede integrar la tecnología en la terapia para mejorar la calidad asistencial y reducir la tasa de fracasos y malas experiencias que los clientes han reflejado en las funestas estadísticas antes mencionadas.

\section{NAVEGANDO LA PRÁCTICA: \\ SIETE COORDENADAS FUNDAMENTALES}

Como hemos señalado antes, fue la llegada del modelo sistémico la que facilitó la creación de los fundamentos de la TP y la consolidó como una disciplina independiente. En la actualidad, otras corrientes como las conductuales, las centradas en las emociones, las basadas en el construccionismo social, o los enfoques multigeneracionales y psicodinámicos han contribuido de forma sustancial al desarrollo de la TP (véase Gurman et al., 2015; para una revisión comparativa de las escuelas). Todos ellos han aportado técnicas y conceptos de las que nutrirse para la práctica con distintos niveles de precisión y sistematicidad.

Mi impresión, no obstante, es que la mayoría de autores de una y otra escuela estarían de acuerdo en que, más allá del debate entre factores comunes y específicos, es necesario establecer algunos puntos clave para orientarse en la práctica, que permitan a los terapeutas navegar con ciertas garantías de supervivencia en el impredecible y movido océano de la TP. Existen ya algunas propuestas de integración, como el protocolo unificado (Christensen, 2010), el modelo de Snyder y Schneider (2002), o el enfoque conductual integrativo de Gurman (2008). Pero sigue siendo esta una cuestión abierta, todavía por abarcar e integrar con la práctica clínica (Sparks y Duncan, 2010).

Mi forma de concebir la práctica de la TP es muy próxima a la forma de vivir una aventura. Es un viaje que se sabe cómo empieza pero no cómo termina; $\mathrm{y}$ en el que, inevitablemente, surgen dificultades, percances y sorpresas de todo tipo. Para lidiar con la incertidumbre, más vale disponer de mapas para orientarse en el 
camino, pero sin olvidar que son las parejas las que recorren el territorio. Empezar una TP supone pues empezar una aventura, especialmente para los miembros de la pareja, que en un momento determinado deciden buscar ayuda externa para resolver sus desencuentros e infortunios. Como acompañantes tenemos el deber ético de proveer las garantías necesarias de seguridad, profesionalidad y confidencialidad. Para ello, el terapeuta debe adoptar una posición epistemológica acerca del ser humano y la naturaleza del cambio. Pero además, necesita disponer de una guía de navegación que le ayude a explorar, junto con las parejas, los territorios amoroso, relacional, íntimo y sexual.

Sin el ánimo de crear una lista exhaustiva y excluyente, lo que aquí presento es una invitación a la práctica desde la práctica. Una lista incompleta de coordenadas para la navegación, basadas en mi propia experiencia, que espero pueda servir de punto de partida para algunos, de síntesis para otros, y objeto de crítica y reflexión para todos los lectores. Por el momento cuento, al menos, siete coordenadas fundamentales que toda TP debería contemplar en su hoja de ruta:

\section{Perspectiva sistémica y evolutiva}

Todos los enfoques modernos de la TP asumen, al menos de forma parcial, una perspectiva sistémica en el sentido de que se asume que no se trabaja con dos individuos independientes entre sí, sino en total interdependencia. La unidad básica de análisis es por tanto el subsistema de pareja como tal, el cual está regulado por ciertas propiedades y sistemas de significado (Feixas, Muñoz, Compañ y Montesano, 2012; Montesano, 2012; Waztlawick, Beavin, y Jackson., 1967). Cualquier acto, discurso, o emoción debe ser contextualizada en su marco relacional.

Asimismo, la perspectiva evolutiva nos advierte de la importancia de entender a las parejas en un proceso de desarrollo continuo, en el que se entrelazan la necesidad de cambiar para adaptarse a los retos impuestos por el ciclo vital y la lucha por preservar la integridad del sistema. Cada etapa del ciclo vital de la pareja implica una serie de desafíos y tareas específicas a las que el sistema debe hacer frente, del mismo modo que cada contexto cultural-relacional enmarca la semántica en la que está inmersa la pareja. Muchas veces las crisis vienen derivadas por dificultades de transición entre una coordenada y la siguiente y la imposibilidad de resignificar el proyecto. Determinar las coordenadas del momento del ciclo vital nos da idea del lugar en que se encuentra la pareja en todas sus dimensiones, afectiva, relacional, familiar, sexual, etc.

\section{Centrada en el presente, mirando hacia el futuro}

Tal y como afirmó Haley (1963) en su artículo seminal Terapia Matrimonial, las parejas son expertas en discutir sobre el pasado. Los psicoterapeutas con experiencia en TP saben bien a lo que se refería Haley con esta idea. Por ello, suelen evitar la espiral de discusiones infructuosas y centran la intervención en ayudar a la pareja en su momento actual del ciclo vital, preparándoles incluso para la etapa 
que este por llegar.

Es cierto que para hacer un adecuado diagnóstico relacional es a menudo preciso bucear en la historia de la familia de origen de cada miembro y en la historia de amor de la pareja. Pero, dicho viaje submarino sólo tiene sentido si está orientado a entender lo que le sucede a la pareja en el presente. En determinadas circunstancias (e.g., rencor, infidelidad), los conflictos del pasado tiene un peso tal en el presente que sólo su disolución permite a la pareja desanclarse para evolucionar hacia un horizonte mejor cualquiera que este sea (incluyendo, por supuesto, la separación). Pero es del todo desaconsejable que el terapeuta se empeñe en ventilar trapos sucios y buscar las causas del problema actual en conflictos pasados.

Si la pareja es capaz de cultivar una relación satisfactoria en el presente, los problemas del pasado probablemente caerán por su propio peso. Como reza la célebre idea de Pittman, los problemas de pareja no se resuelven sino que se disuelven. Así pues, la primera pregunta que todo terapeuta debe hacerse frente a una pareja que le consulta es por qué buscan ayuda ahora. La segunda es hacia dónde vamos después.

\section{Formato de terapia breve}

La TP tiene, por naturaleza, un formato de terapia breve. El número medio de sesiones oscila entre las 8 y las 18 (Doherty y Simmons, 1996). Muchos protocolos de terapia individual han reducido en las últimas décadas la duración de los tratamientos hasta cuotas similares, pero la TP ha sido así desde sus comienzos. Por ejemplo, Gurman y Kniskern (1978) señalaron a finales de los años setenta que la mayoría de los cursos terapéuticos en TP tenían menos de 20 sesiones, una época en la que la terapia individual podía alcanzar cuotas de hasta 600 sesiones (e.g., psicoanálisis). Hoy casi 40 años después, los protocolos de TP siguen siendo breves incluso para problemas resistentes como la depresión crónica (Montesano, Feixas y Eiroa-Orosa, 2013).

Aunque el número de sesiones sea reducido, la terapia puede extenderse en un periodo de tiempo relativamente extenso, teniendo en cuenta el intervalo de tiempo que se deja entre sesiones. La mayoría de los terapeutas coinciden en señalar la importancia de prolongar el espacio entre sesiones una vez formulado el diagnóstico relacional con la intención de que los cambios puedan consolidarse. Las sesiones de seguimiento a medio/largo plazo son muy recomendables, especialmente cuando la pareja ha consultado por infidelidad, algún tipo de violencia o por problemas en su actividad sexual.

Lo que todavía está por dilucidar es cómo y cuándo cambian las parejas, es decir, cuáles son los patrones de cambio más habituales en TP. Esperamos que la investigación nos de pronto algunas respuestas sobre este asunto. No obstante, creo que muchos clínicos estarían de acuerdo conmigo en señalar que los primeros brotes del cambio pueden apreciarse en la fase temprana de la terapia, entre la primera y la tercera sesión, y que hacia la octava sesión el cambio ya suele estar en marcha. 
Por regla general, si a estas alturas de la terapia no se ha producido ningún atisbo de cambio, es señal de que la terapia debe reorientarse o incluso abandonarse. Por supuesto, como toda regla general, falla en el territorio de la particularidad.

\section{Focalizada en los procesos del sistema que mantienen/ disuelven el problema}

Dada la naturaleza breve y centrada en el presente de la TP, para la mayoría de los terapeutas de pareja resulta esencial poder establecer focos concretos que guíen la intervención. Por lo general, los terapeutas de pareja se centran en desvelar y desafiar los patrones relacionales que están ligados al problema (Pinsof, 1995). Estos patrones, entendidos como agentes mantenedores/ disolventes del problema, deben ser formulados tras una adecuado diagnóstico relacional de la pareja.

Una propuesta muy refinada en el panorama nacional para el diagnóstico relacional y la intervención es la de Campo (2014), muy recomendable tanto para terapeutas en formación como para terapeutas experimentados. Otros trabajos internacionales de vanguardia que invitamos a explorar al lector son el de los metamarcos del Instituto de la Familia de Chicago (Breulin, Pinsof, Russell, Lebow, 2011; Pinsof, Breunlin, Russell y Lebow, 2011), o los patrones relacionales del Centro de Terapia Familiar de Calgary (Tomm, St. George, Wulff, y Strong, 2014). Todos ellos son buenas guías para un diagnóstico relacional completo que contemple tanto las dinámicas mantenedoras del problema como las dinámicas de relación constructivas.

\section{Balance a favor de los recursos}

Aunque los patrones relacionales que mantienen el problema acostumbran a ser un foco importante de la intervención terapéutica, una formulación relacional debe dar cuenta, incluso en mayor medida, de las áreas conservadas de la pareja, los recursos con los que cuentan sus miembros para facilitar la creatividad con que una pareja puede transformarse a sí misma. Un patrón disfuncional no equivale a una pareja disfuncional. Los problemas pueden llegar a ser muy persistentes y abarcativos, pero nunca cubren la totalidad de la extraordinaria complejidad que involucra una relación de pareja. Siempre hay excepciones al problema, aunque estén escondidas en la imaginación.

La experiencia clínica muestra una y otra vez que es muy recomendable sino imprescindible que en etapas intermedias de la terapia la arquitectura de las sesiones se focalice significativamente más en reconocer y amplificar las excepciones al problema que en el problema en sí. Para ello, es esencial mantener la balanza a favor de los recursos y fomentar las interacciones positivas, que servirán de termómetro a la pareja para calibrar su relación. Ayudar a las parejas a fomentar, desenterrar, reconocer o experimentar episodios relacionales que producen dinámicas relacionales constructivas (círculos virtuosos), facilita que la pareja pueda reformular su proyecto, disolver tensiones, y madurar la relación, incluso si ello implica la separación. 
Lo que trato de transmitir es que hay que generar una formulación relacional del cambio, que permita a la pareja revisar su proyecto, redefinirse. El cambio puede operar a través de mecanismos muy diversos. Benson, Mcuinn y Christensen (2010) en su esfuerzo integrativo han señalado cinco principios comunes que facilitan el cambio en la TP: (a) Alterar la visión de la pareja de su problema para que sea más contextualizada y diádica, (b) disminuir la conducta emocional disfuncional, (c) elicitar la experiencia emocional interna y evitada, (d) incrementar los patrones de interacción constructivos, y (e) reforzar los cambios obtenidos durante la terapia.

Otros principios comunes no contemplados en la lista pueden ser el restablecimiento de la esperanza, la reparación, la recuperación de espacios de intimidad, o la incorporación de nuevos significados al proyecto de pareja. Sea cual fuere el circuito que se tome, el cambio se construye más en base a lo que ofrecen las excepciones que al discurso del propio problema en sí.

\section{Integra la experiencia personal e interpersonal}

En los párrafos anteriores hemos conceptualizado a la pareja en términos de sistema y hemos recomendado focalizar la intervención en la dinámica relacional tanto positiva como negativa de la pareja. Pero ello no es óbice para tener en cuenta factores de la experiencia intrapsíquica de los miembros de la pareja así como su relación con el patrón de interacción. Más bien todo lo contrario, esta es precisamente la forma de completar el mapa.

La integración entre aspectos de la experiencia personal e interpersonal ha sido una de las últimas áreas de desarrollo de la TP, que ha venido de la mano de la propia evolución del modelo sistémico desde la pragmática a la semántica y la incorporación de otros modelos. La mayoría de protocolos incluyen sesiones individuales dentro del marco de la TP. La secuencia más común consiste en realizar una fase de evaluación primero con la pareja y después con cada miembro, y realizar finalmente una sesión conjunta para negociar el contrato terapéutico. Para favorecer el acuerdo, además de que el problema y la solución se definan en términos relacionales, es del todo preciso dar cuenta de la experiencia subjetiva de cada miembro, y su particular forma de construirse a sí mismo y a la relación.

Una herramienta clínica de gran utilidad que permite trabajar con la interconexión de la experiencia personal e interpersonal es el diagrama circular(Montesano, 2015). Este es un procedimiento para mapear el proceso de construcción en las parejas integrando: a) La dimensión pragmática y la semántica; y (b) los discursos sociales y la familia de origen de cada miembro. Es una herramienta que facilita la formulación del caso, pero además es una intervención en sí misma que favorece el entendimiento interpersonal y la resolución de problemas. El diagrama circular permite a su vez examinar el cambio durante la terapia, mantener una práctica reflexiva, o ser utilizado en supervisión. 


\section{Focalizada en el contexto relacional natural}

Ya hemos anunciado antes el valor ecológico que el setting de la TP confiere a la intervención terapéutica, al incidir sobre el contexto relacional natural, así como sus implicaciones para el desarrollo de la alianza terapéutica. Como ha sido ampliamente demostrado en la investigación, la intervención en terapia individual, sea del signo que sea, está fuertemente sustentada sobre la relación terapéutica ente el terapeuta y el cliente. En efecto la alianza terapéutica, ha demostrado tener una gran capacidad predictiva de los resultados de la terapia, por encima de cualquier otro aspecto técnico.

Sin embargo, en el contexto de la TP, la relación ente el terapeuta y los clientes es mucho menos intensa y central, sin apenas influencia sobre los resultados de la terapia. El cambio no está vehiculizado por esta interacción sino por la relación entre los miembros de la pareja. Por ello, el terapeuta, una vez garantizados unos mínimos de alianza con ambos, debe focalizar sus esfuerzos en incrementar el grado de alianza entre los miembros de la pareja, pues este es el contexto relacional donde el cambio se da (Escudero, 2009, Montesano et al., 2014; Montesano e Izu, 2015). Si bien en terapia individual se pretende, por ejemplo, generar una experiencia emocional correctiva que sea generalizada después al contexto natural del cliente, en TP se incide directamente sobre este, in situ. Seguramente este sea también un motivo por el cual la terapia tiende a ser breve, pues no es necesario desarrollar una relación profunda con los miembros de la pareja por separado.

En cualquier caso, focalizar en la alianza intra-sistema, es en verdad una manera de enfocar en el patrón relacional y en el reposicionamiento sobre el mismo. Este reposicionamiento puede implicar diferentes niveles de profundidad. En algunos casos, será suficiente con sondear las excepciones ya existentes y potenciarlas. En otros, habrá que bucear hasta territorios más profundos de la jerarquía de poder de la pareja, de las familias de origen, o de secretos todavía por desvelar. De una manera u otra siempre el viaje comienza por ayudar a la pareja a mirarse a sí misma, y que cada miembro defina su posición en los ejes de coordenadas descritos. ¡Buen viaje!

\section{Referencias bibliográficas}

Baucom, D. H., Belus, J. M., Adelman, C. B., Fischer, M. S. y Paprocki, C. (2014). Couple based interventions for psychopathology: A renewed direction for the field. Family Process, 53(3), 445-461.

Breunlin, D. C., Pinsof, W., Russell, W. P. y Lebow, J. (2011). Integrative problemcentered metaframeworks therapy I: core concepts and hypothesizing. Family Process, 50, 293-313.

Campo, C. (2014). La terapia de pareja en la práctica clínica: Un modelo de diagnóstico e intervención (pp. 251278). En R. Medina, E. Laso y E. Hernández, (Eds.), Pensamiento sistémico: Nuevas perspectivas y contextos de intervención. México: Litteris.

Christensen, A. (2010). A unified protocol for couple therapy. In K. Hahlweg, M. Grawe-Gerber y D. H. Baucom (Eds.), Enhancing couples: The shape of couple therapy to come. Göttingen, Germany: Hogrefe.

Christensen, A., Atkins, D. C., Berns, S., Wheeler, J., Baucom, D. y Simpson, L. E. (2004). Traditional versus 
integrative behavioral couple therapy for significantly and chronically distressed married couples. Journal of Consulting and Clinical Psychology, 72, 176-191.

Darwiche, J. y de Roten, Y. (2015). Couple and Family Treatments: Study Quality and Level of Evidence. Family Process, 54(1), 138-159.

Doherty, W.J. y Simmons, D.S. (1996). Clinical practice patterns of marriage and family therapists: A national survey of therapists and their clients. Journal of Marital and Family Therapy, 22, 9-25.

Escudero, V. (2009). La creación de la alianza terapéutica en la terapia familiar. Apuntes de Psicología, 27 (2-3), 247-259.

Feixas, G., Muñoz, D., Compañ, V. y Montesano, A. (2012). El modelo sistémico en la intervención familiar. Colección OMADO, Depòsit digital de la Universitat de Barcelona, Creative Comuns Catalunya http:// hdl.handle.net/2445/31584

Gurman, A.S. y Kniskern, D.P. (1978). Research on marital and family therapy: Progress, perspective, and prospect (pp. 817-901). In S.L. Garfield y A.E. Bergin (eds.), Handbook of psychotherapy and behavior change (2nd ed.). New York: John Wiley y Sons.

Gurman, A. S. (2008). Integrative couple therapy: A depth-behavioral approach. In A. S. Gurman (Ed.), Clinical handbook of couple therapy (4th ed.). New York: Guilford press.

Gurman, A. S. y Fraenkel, P. (2002). The history of couple therapy: A millennial review. Family Process, 41, 199_ 260.

Gurman, A. S., Lebow, J. y Snyder, D. K. (2015). Clinical handbook of couple therapy (5th ed.). New York, NY: Guilford Press.

Haley, J. (1963). Marriage therapy. Archives of General Psychiatry, 8, 213-234.

Leff, J., Vearnals, S., Brewin, C., Wolff, G., Alexander, B., Asen, E., Dayson, D., Jones, E., Chisholm, D. y Everitt, B. (2000). The London Depression Intervention Trial. Randomised Controlled Trial of Antidepressants v. Couple Therapy in the Treatment and Maintenance of People with Depression Living with a Partner: Clinical Outcome and Costs. British Journal of Psychiatry, 177, 95-100.

Montesano, A. (2015). The Circular Diagram in couple therapy: A guiding map for the therapeutic process. Manuscrito sometido a publicación

Montesano, A. (2012). La perspectiva narrativa en terapia familiar sistémica. Revista de Psicoterapia, 89, 5-50.

Montesano, A. y Izu, S. (2015). Análisis del proceso terapéutico en un caso de terapia de pareja para la depresión: Cómo lidiar con una alianza intra-sistema problemática. Manuscrito sometido a publicación.

Montesano, A., Feixas, G. y Eiroa-Orosa, F. J. (2013, September). Systemic couple therapy for depression: efficacy of a short-term intervention protocol. Paper in panel: How Effective are Short Term Interventions? State of Research. Society for Psychotherapy Research- UK Chapter Annual meeting. Oxford, United Kingdom.

Montesano, A., Feixas, G., Muñoz, D. y Compañ, V. (2014). Systemic Couple Therapy for Dysthymia: an evidencebased case study. Psychotherapy, 51, 30-40.

Orlinsky, D. E. y Ronnestad, M. H. (2005). How psychotherapists develop: A study of therapeutic work and professional growth. Washington. DC: American Psychological Association.

Pinsof, B. (1995), Integrative Problem Centered Therapy. New York: Basic Books.

Pinsof, W., Breunlin, D. C., Russell, W. P. y Lebow, J. (2011). Integrative problem centered metaframeworks therapy II: planning, conversing, and reading feedback. Family Process, 50, 314-336

Selekman, M. y Beyebach, M. (2013). Changing self-destructive habits. Pathways to solutions with couples and families. New York: Routledge.

Seligman, M.E. (1995). The effectiveness of psychotherapy: The Consumer Reports study. American Psychologist, 50, 965-974.

Shadish, W. R. y Baldwin, S. A. (2005). Effects of behavioral marital therapy: A meta-analysis of randomized controlled trials. Journal of Consulting and Clinical Psychology, 73(1), 6-14.

Snyder, D. K. y Halford, W. K. (2012). Evidence-based couple therapy: Current status and future directions. Journal of Family Therapy, 34, 229-249.

Snyder, D. K. y Schneider, W. J. (2002). Affective reconstruction: A pluralistic, developmental approach. In A. S. Gurman y N. S. Jacobson (Eds.), Clinical handbook of couple therapy (3rd ed.) (pp. 151-179). New York, NY: Guilford Press.

Sparks, J. A. y Duncan, B. L. (2010). Common factors in couple and family therapy: Must all have prizes? In B. L. Duncan, S. C. , Miller., B. E. Wampold y M. A. Hubble (Eds.), Heart and soul of change: Delivering what works in therapy (2nd ed.). (pp. 357-392). Washington, DC: American Psychological Association.

Tomm, K., St. George, S., Wulff, D. y Strong, T. (2014). Patterns in interpersonal interactions: Inviting relational understandings for therapeutic change. New York: Routledge. 
Watzlawick, P., Beavin, J. H. y Jackson, D.D. (1967). Pragmatics of Human Communication. New York: Norton. Whisman, M. A., Johnson, D. P., Be, D. y Li, A. (2012). Couple-Based Interventions for Depression. Couple and Family Psychology: research and practice, 3, 185-198. 